\title{
Colorectal cancer liver metastases: management and five-year survival in a South African patient cohort
}

\author{
M Brand, ${ }^{1,2}$ (iD) P Gaylard, ${ }^{3}$ (D) $\mathrm{J} \mathrm{Ramos}^{4}(\mathrm{D}$ \\ ${ }^{1}$ Department of Surgery, University of Pretoria, South Africa \\ ${ }^{2}$ School of Physiology, University of the Witwatersrand, South Africa \\ ${ }^{3}$ Data Management and Statistical Analysis, South Africa \\ ${ }^{4}$ WITS Donald Gordon Medical Centre; University of the Witwatersrand, South Africa
}

Corresponding author, email: rispah.chomba@wits.ac.za

Background: Approximately $25 \%$ of patients with colorectal cancer (CRC) will be diagnosed with CRC liver metastases (CRCLM) during the course of their disease. No data regarding CRCLM presentation, management and survival outcomes has been published from either the private or public health care sectors in South Africa. This study aimed to address this deficit, reporting on a private sector cohort.

Methods: A retrospective review of a private health care funder's database from 1 January 2008 to 31 December 2015 was performed. ICD-10 diagnosis codes were used to identify CRC and CRCLM. Procedure codes assigned to hospital admissions were used to identify the type of surgical treatment. Chemotherapy was identified by the WHO Anatomical Therapeutic Chemical classification system of medicines. Treatment patterns were assessed and five-year overall survival (OS) was calculated. Survival was estimated using the Kaplan-Meier method, and Cox proportional-hazards regression was used for between group survival comparisons.

Results: Six hundred and one (601) of 3412 patients presenting with CRC (17.6\%) were diagnosed with CRCLM at presentation or during the follow-up period. Sixty patients with CRCLM (10.0\%) underwent resection of the primary CRC and liver resection for metastases, 281 (46.8\%) underwent CRC resection only, 180 (30\%) received chemotherapy only, and 47 (7.8\%) received no treatment. Five-year OS for these groups were $57.3 \%, 15.6 \%, 9.8 \%$ and $0 \%$ respectively. Conclusion: Five-year OS of the various CRCLM treatment pathways in a South African private sector population compares to results published in international series. However, a smaller proportion of patients with CRCLM underwent liver resection, compared to international studies.

\section{Background}

In South Africa colorectal cancer (CRC) is the fourth most common cancer among both men and women with crude annual incidences of 7.17/100 000 for men and 5.80/100 000 for women. ${ }^{1}$ Approximately $25 \%$ of CRC patients will show to have liver metastases, apparent at diagnosis of the primary tumour or detected during the course of the disease. ${ }^{2,3}$ Previously, selection of patients with CRC liver metastases (CRCLM) for liver resection was based on a number of tumour-related factors, including size, number and segmental distribution. ${ }^{4}$ However, recent better understanding of tumour biology, advances in oncologic treatment and interventional radiology techniques, and development of novel surgical techniques have allowed for expanding indications for potentially curative resections for CRCLM. ${ }^{5}$ This study was undertaken to assess the management of CRCLM in a privately insured South Africa patient cohort, as well as survival statistics for the various treatment groups.

\section{Methods}

\section{Study design and data collection}

A retrospective review of a private health care funder's database from 1 January 2008 to 31 December 2015 was performed. Consent was obtained for de-identified data from the principal officer of the private health care funder. Patients enrolled on the scheme's oncology benefit for the treatment of CRC in the period 1 January 2008 to 31 December 2015 were included in the study. ICD-10 diagnosis codes were used to identify patients with CRC and CRCLM. Comprehensive data was extracted to allow at least a oneyear follow-up to ensure inclusion of data on post-surgery oncologic management. For this tables, based on claims and clinical information for active members of the medical scheme, developed by the medical scheme for analysis where the table development included data validation were used. ${ }^{6}$ The timing and type of all surgical treatments relevant to $\mathrm{CRC}$ and CRCLM were identified, using procedure codes for resection assigned to hospital admissions. Chemotherapy 
was identified by the WHO Anatomical Therapeutic Chemical classification system of medicines. Patients were excluded if they were younger than 18 years old. Data on metastases at sites other than the liver were not collected and are therefore not considered in analyses.

Patients with liver metastases were assessed according to treatment group, with/without liver resection and with/ without perioperative chemotherapy. Synchronously detected metastases were defined as detected before or at the time of surgery for the primary tumour or within a month of diagnosis of the primary in non-operated patients. Date of withdrawal from the medical scheme was used as a surrogate for all-cause mortality and ongoing contribution to the scheme was interpreted as a patient still being alive.

\section{Statistical analysis}

Survival was estimated using the Kaplan-Meier method and Cox proportional-hazards regression was used for between group survival comparisons. A 5\% significance level was used. Data analysis was performed on SAS version 9.4 for Windows (SAS Institute 2016).

\section{Results}

Of 3412 patients with CRC, 601 (17.6\%) were diagnosed with liver metastases during the study period. This group forms the basis of this study the treatment pathways of which are depicted in Figure 1. The median age of the CRCLM patients was 63 years (range 25-93 years) and $59.4 \%$ were males. Thirty-three patients $(5.5 \%)$ were excluded due to incomplete data, i.e. the exact treatment could not be established. Of the remaining patients 60 $(10.0 \%)$ underwent resection of the primary CRC and liver metastases, 281(46.8\%) underwent CRC resection only, $180(30.0 \%)$ received chemotherapy only and $47(7.8 \%)$

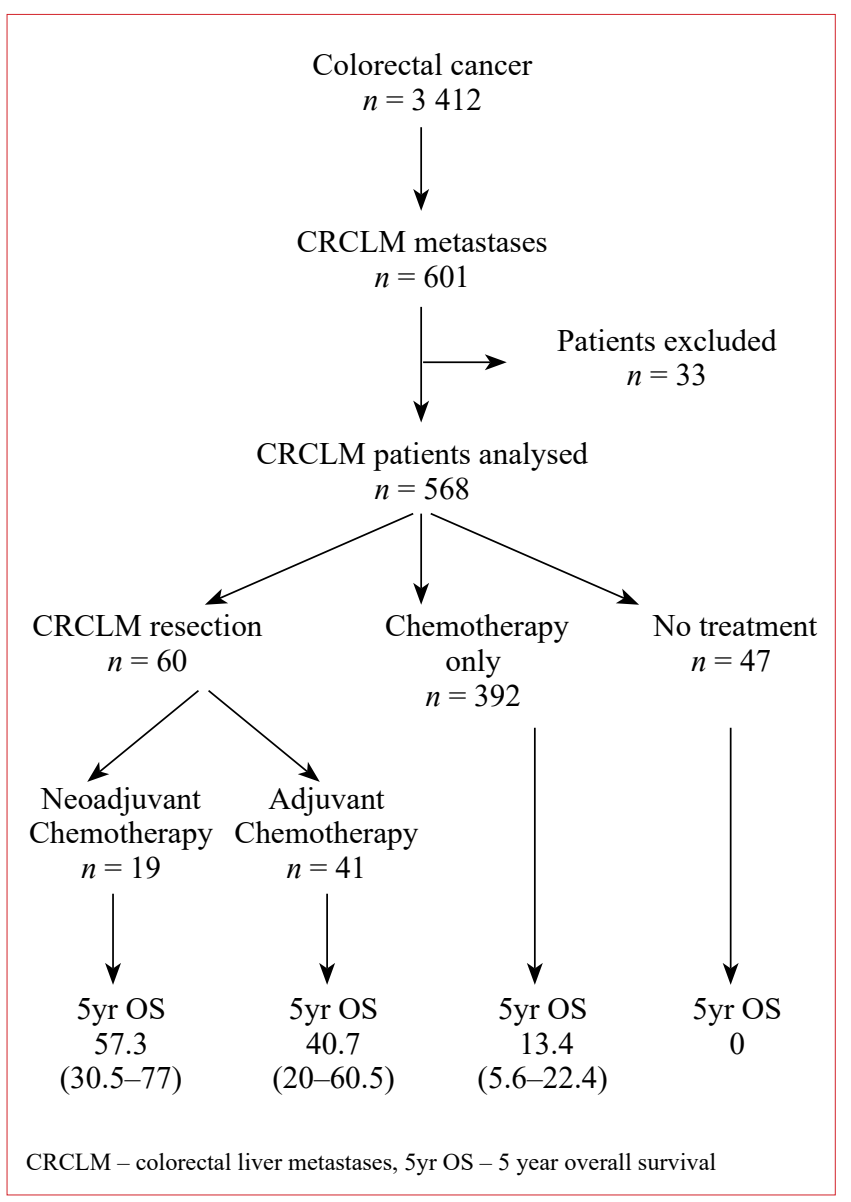

Figure 1: Summary of patient treatment pathways

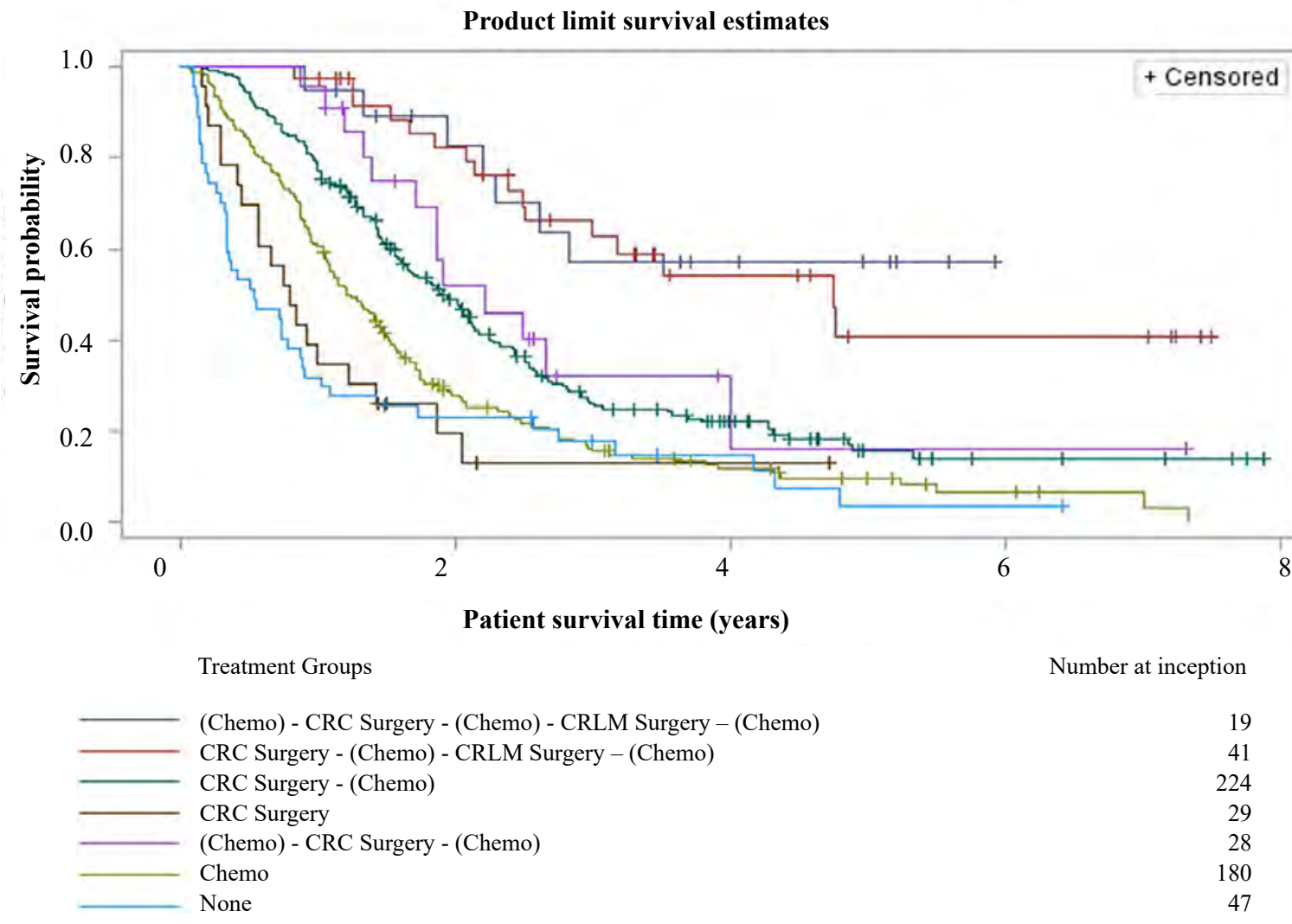

Figure 2: Five year overall survival for various CRCLM treatment pathways 
received no treatment. In patients that underwent resection of liver metastases, CRC resection was performed before liver resection in $56(93.3 \%)$ and liver resection before the CRC resection in $4(6.7 \%)$.

Combinations of folinic acid (FOL) and fluorouracil (F), irinotecan (IRI) and oxaliplatin (OX) were administered to 338 patients $(56.1 \%)$. Of these, $50(14.8 \%)$ underwent CRCLM resection, 23 (18.9\%) following FOLFOX, $4(4.8 \%)$ following FOLFIRI and $23(17.3 \%)$ following FOLFIRINOX. The use of chemotherapy, regardless of regimen, combined with resection of CRCLM, significantly improved five-year OS compared to chemotherapy without resection of CRCLM (FOLFOX $p<0.0001$, FOLFIRI $p=0.033$ and FOLFOX and FOLFIRI $p=0.014$ ).

Biologic agents (bevacizumab or cetuximab) with chemotherapy were used in $236(46.6 \%)$ patients. However, their use was heterogenous and could not be included in the analyses. Five-year OS for no treatment, chemotherapy only and chemotherapy with surgical resection was $0 \%, 9.8 \%$ and $41-57 \%$ respectively. Survival data is presented in Figure 2 (Kaplan-Meier graph of overall survival per treatment cohort).

\section{Discussion}

The median overall survival of patients with untreated CRCLM is less than one year. ${ }^{7}$ With chemotherapy alone 1 -year survival is $5-15 \%$ and following liver resection 5-year overall survival is $31-45 \%{ }^{8}$ This audit of a South African private health care funder's database demonstrates, similar to international trends, that 5-year OS for no treatment, chemotherapy only and chemotherapy with surgical resection is $0 \%, 10 \%$ and $41-57 \%$. However, the overall incidence of CRCLM is lower than reported in international series. This may be due to underreporting of CRCLM, nonoptimal follow-up or incomplete 5-year survival data. It also appears as if fewer CRCLM are treated surgically than in published series. ${ }^{9}$

The survival estimates were capped at five years, beyond which there were too few patients at risk for reliable estimates. Resection of CRCLM has been shown to result in a significant improvement in 5-year OS of 55\% compared to $15 \%$ with no resection. ${ }^{9,10}$ One of the most significant advances of CRCLM management has been the advent of modern cytotoxic agents that may result in conversion of irresectable into resectable disease. ${ }^{11}$ Liver resection following conversion chemotherapy therapy is associated with similar overall survival compared to resected initially resectable liver metastases. ${ }^{12}$ Modern treatment strategies now include neoadjuvant chemotherapy with or without monoclonal antibodies (biologic agents), sequential surgery, synchronous surgery of primary and liver metastases and the liver resection first approach.

In the reported patient cohort neoadjuvant chemotherapy resulted in a significant survival benefit compared to palliative chemotherapy without surgery. However, we cannot comment on the staging of disease in the chemotherapy only group. Overall, 5-year survival of surgical resection of the primary cancer and liver metastases followed by chemotherapy was similar to chemotherapy followed by surgical resection. The majority of synchronous disease was managed with a primary cancer resection preceding the CRCLM resection as a second procedure.

\section{Chemotherapy}

The probability that liver resection may offer long-term survival to patients with multiple CRCLM is higher in patients where the metastatic disease is controlled by neoadjuvant chemotherapy prior to surgery. Five-year OS in patients who underwent liver resection following neoadjuvant chemotherapy with an objective tumour response compared to stable disease, or tumour progression was $37 \%, 30 \%$ and $8 \%$ respectively. ${ }^{13}$ The type of neoadjuvant chemotherapy agents used does not influence the outcomes provided that complete resection of all liver metastases is achieved. However, the shorter the course of neoadjuvant chemotherapy and the fewer the number of chemotherapy lines administered, the lower the postoperative morbidity and longer overall survival; ${ }^{13,14}$ the threshold appears to be five cycles. ${ }^{15}$ Our data indicates that with both FOLFOX and FOLFIRI chemotherapy, the survival of those who received surgical resection of either the primary CRC with or without CRCLM resection was better than those who did not. There was no significant difference in overall survival with the use of either FOLFOX or FOLFIRI.

\section{Biologics}

Chemotherapy with molecular target agents has provided further improvement in tumour response rates. ${ }^{16}$ The value of biologic agents in relation to liver resection seems to be in the neoadjuvant setting, as chemotherapy with the addition of bevacizumab has been shown to have no impact on either recurrence free survival or overall survival in the adjuvant setting following complete resection of colorectal liver metastases. ${ }^{17}$ This tendency is seen in the presented data. However, the routine use of neoadjuvant chemotherapybiologic agent is controversial, and guidelines currently only recommend this combination for conversion therapy. ${ }^{18}$ The concurrent use of bevacizumab with oxaliplatin-based chemotherapy as a first-line therapy did not result in a significantly increased response rate or resection rate of liver metastases; ${ }^{19}$ more recent studies have demonstrated a response rate of $30-81 \%$ in anti-VEGF treated CRCLM patients, similar to the $30-73 \%$ in chemotherapy only treated patients (including quadruple chemotherapy regimens). ${ }^{20,21}$

\section{Multidisciplinary team assessment}

In patients with CRCLM the most important factor that affects patient prognosis is the resectability of the metastatic lesions. As a result of the increasing complexity of CRCLM management, patients are often undertreated unless a liver surgeon is involved early in the decision-making process. Discussion in a multidisciplinary team (MDT) composed of appropriate specialists as early as possible in a patient's management algorithm is crucial. ${ }^{22}$ A Swedish study, where MDT discussion is a national requirement for each patient with $\mathrm{CRC}$, showed that when no liver surgeons were on these teams only $17.8 \%$ of liver metastases were resected, a percentage below international norms. ${ }^{23} \mathrm{~A}$ British study compared the treatment decisions determined initially by non-liver surgeons and compared them to liver surgeons' opinions; the liver surgeons felt that $63 \%$ of the cases deemed not resectable by the MDT were resectable. ${ }^{23}$ An American study found that referral to a liver surgeon was associated with a 5 -year OS of $33 \%$ compared with $8 \%$ in patients who were not. ${ }^{24}$ Hence, patients with CRCLM that are managed without the involvement of a specialist liver 
MDT may lead to some patients being denied potentially curative treatments. This study was not able to determine whether or not South African CRCLM patients are routinely discussed in an MDT; however, from international literature MDTs should be the norm for these patients.

There are limitations in this study. Data was retrieved from a single private health care funder's database that represents $53 \%$ of South Africans with health insurance. The database is primarily designed for health economic data capture with limited clinical information, thus study results are based on treatments claimed from the medical scheme. In other words, treatments that members did not claim from the scheme are not included. Examples may include treatment received as part of a clinical trial, self-funded medication or treatment received from state facilities. Furthermore, access to certain treatment modalities is dependent on the patient's medical aid plan, for example access to biologic medications is not available to all patients. As a result of the paucity of clinical information, only treatment patterns are reported. Follow-up in some patients was only one year which may have resulted in under-reporting of CRCLMs. Differences observed in the reported cohort compared to international studies are more likely the result of incomplete data than the disease being different in South African patients. The results of this study cannot be extrapolated into the public health care sector as the treatment of the disease seemingly differs significantly with regards to disease presentation as well as access to treatment such as chemotherapy and biologic agents. The survival estimates and Kaplan-Meier plot are limited by the fact that a surrogate for all-cause mortality derived from secondary medical aid administrative data and not primary clinical data was used. Therefore, we could not adjust for covariates and there may be missing information. Furthermore, not accounting for patients with extrahepatic metastases is an additional shortcoming in the study.

\section{Conclusion}

We have demonstrated that 5-year OS is improved with the use of chemotherapy and better following surgical resection of CRCLM compared to chemotherapy alone. All patients with CRCLM should be managed in an MDT, either with neoadjuvant or adjuvant chemotherapy. The use of biologic agents without CRCLM resection does not seem to improve overall survival.

\section{Acknowledgements}

Discovery Health, specifically the Clinical Policy Unit, for their assistance with data extraction and management.

\section{Conflict of interest}

The authors declare no conflict of interest.

\section{Funding source}

Wits Donald Gordon Medical Centre, University of the Witwatersrand, for funding the statistical analysis of the data.

\section{Ethical approval}

Ethics approval was granted by the University of the Witwatersrand Human Research Ethics Committee (M141027).

\section{ORCID}

M Brand (D) https://orcid.org/0000-0001-8285-3880

P Gaylard (iD https://orcid.org/0000-0002-0055-9168

J Ramos (i) https://orcid.org/0000-0003-1341-7684

\section{REFERENCES}

1. Cancer Association of South Africa. 2012 National Cancer Registry. Available from: http://www.cansa.org. za/files/2017/03/SA-National-Cancer-Registry-2012-webFeb-2017.pdf. Accessed 8 April 2017.

2. Van Cutsem E, Nordlinger B, Adam R, et al. Towards a pan-European consensus on the treatment of patients with colorectal liver metastases. Eur J Cancer. 2006;42(14):221221.

3. Hackl C, Neumann P, Gerken M, et al. Treatment of colorectal liver metastases in Germany: a ten-year population-based analysis of 5772 cases of primary colorectal adenocarcinoma. BMC Cancer. 2014;14:810.

4. Altendorf-Hofmann A, Scheele J. A critical review of the major indicators of prognosis after resection of hepatic metastases from colorectal carcinoma. Surg Oncol Clin N Am. 2003;12(1):165-92.

5. Manfredie S, Lepage C, Hatem C, et al. Epidemiology and management of liver metastases from colorectal cancer. Ann Surg. 2006;244(2):254-9. http://doi.org/10.1097/01. sla.0000217629.94941.cf

6. Brand M, Gaylard P, Ramos J. Colorectal Cancer in South Africa: an assessment of disease presentation, treatment pathways and 5-year survival. SAMJ. 2018;108(2):118-22.

7. Jemal A, Bray F, Center MM, et al. Global cancer statistics. CA Cancer J Clin. 2011;61:69-90.

8. Nathan H, De Jong MC, Pulitano C, et al. Conditional survival after surgical resection of colorectal liver metastasis: an international multi-institutional analysis of 949 patients. J Am Coll Surg. 2010;210:755-66.

9. Lemke J, Cammerer G, Ganser J, et al. Survival and prognostic factors of colorectal liver metastases after surgical and nonsurgical treatment. Clin Colorectal Cancer. 2016 Dec;15(4):e183-e192. http://doi.org/10.1016/j. clcc.2016.04.007.

10. Brouquet A, Abdalla EK, Kopetz S, et al. High survival rate after two-stage resection of advanced colorectal liver metastases: response-based selection and complete resection define outcome. J Clin Oncol. 2011;29(8):1083-90.

11. Poston G, Adam R, Vauthey JN. Downstaging or downsizing: time for a new staging system in advanced colorectal cancer? J Clin Oncol. 2006;24(18):2702-6.

12. Morris EJA, Forman D, Thomas JD, et al. Surgical management and outcomes of colorectal cancer liver metastases. Br J Surg. 2010;97:1110-8.

13. Adam R, Barroso E, Laurent C, et al. Impact of the type and modalities of preoperative chemotherapy on the outcome of liver resection for colorectal metastases. J Clin Oncol. 2011;29(15 Suppl):3519.

14. Okuno M, Hatano E, Kasai Y, et al. Feasibility of the liverfirst approach for patients with initially unresectable and not optimally resectable synchronous colorectal liver metastases. Surg Today. 2016;46(6):721-8.

15. Karoui M, Penna C, Amin-Hashem M, et al. Influence of preoperative chemotherapy on the risk of major hepatectomy for colorectal liver metastases. Ann Surg. 2006;243(1):1-7.

16. Lam VW, Spiro C, Laurence JM, et al. A systematic review of clinical response and survival outcomes of downsizing 
systemic chemotherapy and rescue liver surgery in patients with initially unresectable colorectal liver metastases. Ann Surg Oncol. 2012;19:1292-1301.

17. Turan N, Benekli M, Koca D, et al. Adjuvant systemic chemotherapy with or without bevacizumab in patients with resected liver metastases from colorectal cancer. Oncology. 2013;84(1):14-21.

18. Van Cutsem E, Cervantes A, Adam R, et al. ESMO consensus guidelines for the management of patients with metastatic colorectal cancer. Ann Oncol. 2016;27(8):1386-422.

19. Saltz LB, Clarke S, Rubio E, et al. Bevacizumab in combination with oxaliplatin-based chemotherapy as first-line therapy in metastatic colorectal cancer: a randomized phase III study. J Clin Oncol. 2008;26:2013-19.

20. Uetake $H$, Yasuno $M$, Ishiguro $M$, et al. A multicenter phase II trial of mFOLFOX6 plus bevacizumab to treat liver-only metastases of colorectal cancer that are unsuitable for upfront resection (TRICC0808). Ann Surg Oncol. 2015;22:908-15.

21. Ychou M, Rivoire M, Thezenas S, et al. A randomized phase II trial of three intensified chemotherapy regimens in firstline treatment of colorectal cancer patients with initially unresectable or not optimally resectable liver metastases. The METHEP trial. Ann Surg Oncol. 2013;20:4289-97.

22. Jones RP, Vauthey JN, Adam R, et al. Effect of specialist decision-making on treatment strategies for colorectal liver metastases. Br J Surg. 2012;99(9):1263-9.

23. Norén A, Eriksson HG, Olsson LI. Selection for surgery and survival of synchronous colorectal liver metastases; a nationwide study. Eur J Cancer. 2016;53:105-14.

24. Ahmad A, Reha J, Somasundar P, Espat NJ, Katz SC. Predictors of surgical non-referral for colorectal liver metastases. J Surg Res. 2016;205(1):198-203. 